\title{
Dimensionamento Amostral para Avaliação de Altura e Diâmetro de Plantas de Timbaúva
}

\author{
Alberto Cargnelutti Filho ${ }^{1}$, Maristela Machado Araujo믈 Ezequiel Gasparin ${ }^{1}$, \\ Douglas Rodrigo Becker Foltz ${ }^{1}$
}

${ }^{1}$ Universidade Federal de Santa Maria - UFSM, Santa Maria/RS, Brasil

\begin{abstract}
RESUMO
O objetivo do estudo foi estimar o tamanho de amostra para avaliar a altura de planta $(\mathrm{H})$, o diâmetro do coleto (D) e a relação altura de planta/diâmetro do coleto (HD) de timbaúva (Enterolobium contortisiliquum) e verificar a variabilidade do tamanho de amostra entre tubetes, doses de fertilizante e idades de avaliação. Foram avaliados dez tratamentos, formados pela combinação de tamanhos de tubetes (110 e $\left.180 \mathrm{~cm}^{3}\right)$ e doses de fertilizante $\left(0,3,5,7\right.$ e $9 \mathrm{~g} \mathrm{~L}^{-1}$ de substrato), no delineamento em blocos, com quatro repetições. Em cinco idades foram determinadas H, D e HD. $\mathrm{O}$ tamanho de amostra para H, D e HD é dependente da idade de avaliação. Para os tamanhos de tubetes, doses de fertilizante e idades de avaliação, 22 plantas por unidade experimental (bandeja) são suficientes para estimar a média dessas características, para erro de estimação de $10 \%$ da média, com grau de confiança de $95 \%$.
\end{abstract}

Palavras-chave: Enterolobium contortisiliquum, número de plantas, amostragem.

\section{Sample Size for Height and Diameter Evaluation of Timbauva Plants}

\begin{abstract}
The objective was to estimate the sample size to evaluate height (H), collar diameter (D) and relation height/collar diameter (HD) of timbauva plants (Enterolobium contortisiliquum) and to verify its variability among tubes, dosages of fertilizer and ages of evaluation. Ten treatments were organized in blocks designed with four replication and formed by the combination of sizes of tubes $\left(110\right.$ and $\left.180 \mathrm{~cm}^{3}\right)$ and dosages of fertilizer $\left(0,3,5,7 \mathrm{e} 9 \mathrm{~g} \mathrm{~L}^{-1}\right.$ of substrate). The $\mathrm{H}, \mathrm{D}$ and $\mathrm{HD}$ variables were evaluated in five ages. The sample size to evaluate $\mathrm{H}, \mathrm{D}$ and $\mathrm{HD}$ is dependent on age evaluation. For the tubes, dosages of fertilizer and ages of evaluation, 22 plants per experimental unit (pot) are enough to predict the plants variables, with estimation error equal to $10 \%$ of estimated average, with a reliable rating of $95 \%$.
\end{abstract}

Keywords: Enterolobium contortisiliquum, number of plants, sampling.

\section{INTRODUÇÃO}

Enterolobium contortisiliquum (Vell.) Morong (Fabaceae), conhecida popularmente como timbaúva, tamboril ou orelha-de-macaco, possui madeira adequada para a confecção de canoas de um tronco inteiro (Backes \& Irgang, 2009). Essa espécie, de acordo com
Carvalho (2003), é utilizada para a confecção de chapas de compensado, de gamelas e de brinquedos, devido a sua madeira de baixa densidade. Também é usada em arborização urbana e para a restauração de áreas, em razão de seu rápido crescimento inicial. Apesar dessa 
ampla utilização, há carência de informações sobre a produção de mudas dessa espécie, o que pode causar insucesso de sua propagação e cultivo (Araújo \& Sobrinho, 2011).

Identificar as condições químicas ideais do substrato, ou seja, aquelas favoráveis para a produção de mudas de espécies arbóreas é importante para a implantação de povoamentos com elevada qualidade e produtividade (Wendling et al., 2007). Os substratos, geralmente apresentam baixo teor de nutrientes, porém são adequados quanto a suas características físicas e estruturais. Assim, a adição de fertilizantes ao substrato é a principal fonte de nutrientes para a planta (Oliet et al., 1999). Existem fertilizantes inorgânicos, para o uso em viveiros, e são distintos quanto à matéria-prima, a quantidade de nutrientes e os mecanismos de liberação (Jacobs \& Landis, 2009). Os fertilizantes de liberação lenta e controlada fornecem nutrientes às plantas, por determinado tempo, sincronizando a demanda com a disponibilidade no substrato (Valeri \& Corradini, 2005).

O tamanho e o volume do recipiente influenciam na morfologia e na qualidade de mudas florestais. O tamanho ótimo de um recipiente depende da espécie, do porte final da planta, da densidade de crescimento, do tempo de permanência na fase de produção e do meio de crescimento (Luna et al., 2009). O volume interfere na quantidade de raízes que a planta pode produzir e também no incremento radicular após o plantio a campo (Ritchie et al., 2010).

Nos experimentos conduzidos em viveiro florestal, é importante avaliar, com a precisão desejada pelo pesquisador, características como a altura de planta, $o$ diâmetro do coleto e, consequentemente, a relação altura de planta/diâmetro do coleto durante o crescimento das plantas. Na determinação da qualidade, são utilizadas características que se baseiam nos aspectos fenotípicos, denominados morfológicos, ou nos internos das plantas, denominados fisiológicos (Gomes \& Paiva, 2008). As primeiras são usadas com maior frequência, sendo a altura e o diâmetro do coleto as duas características comumente avaliadas (Haase, 2008).

As plantas de menor altura e maior diâmetro são preferíveis para sítios áridos, enquanto as mais altas são adequadas para os locais onde há alto nível de mato-competição ou predação por animais, podendo indicar genética superior (Haase, 2007). O diâmetro do coleto é a variável que melhor prediz o desempenho no pós-plantio, indicando a qualidade das plantas, porém ocorrem variações para cada espécie e condições de plantio (Ritchie et al., 2010). O valor da divisão dessas duas características indica a robustez da planta, expressando o equilíbrio de crescimento da planta, sendo que menores relações representam maior capacidade de sobrevivência e estabelecimento das plantas no campo (Gomes \& Paiva, 2004).

Nesse contexto, estimativas confiáveis de variáveis morfológicas, facilmente coletadas no campo, como altura e o diâmetro do coleto, permitem melhor planejamento e êxito no pós-plantio, sendo que, para isso, é importante dimensionar o número de plantas que devem ser avaliadas. Foi constatado por Cargnelutti Filho et al. (2012) que o tamanho de amostra (número de plantas) para avaliar a altura de planta, o diâmetro do coleto e a relação altura de planta/diâmetro do coleto de mudas de canjerana (Cabralea canjerana (Vell.) Mart.) é dependente da idade de avaliação. Para esse caso específico, com base na variabilidade dessas características entre as plantas usadas como amostragem-piloto, os autores verificaram que, para os tamanhos de tubetes, tipos de substratos e idades de avaliação estudados, 18 plantas por bandeja foram suficientes para estimar a média de altura de planta, de diâmetro do coleto e de relação altura de planta/diâmetro do coleto de mudas de canjerana, para um erro de estimação igual a $10 \%$ da média estimada, com grau de confiança de $95 \%$.

Embora os resultados apresentados na literatura especializada não possam ser generalizados, pois foram obtidos com base na variabilidade dos dados de uma situação específica de cada experimento, são úteis como referência para o dimensionamento amostral de ensaios semelhantes e cujo pesquisador não possui informação sobre a variabilidade dos dados. Assim, o tamanho de amostra ideal para Eucalyptus saligna Sm., obtido em um estudo em viveiro florestal, com oito bandejas, cada uma contendo 96 plantas, foi de 26 plantas por tratamento, para uma diferença mínima significativa de $10 \%$, e de nove plantas por tratamento, quando a diferença foi de $20 \%$ para a variável diâmetro do coleto. Para a variável altura de planta, o tamanho ideal foi de 23 plantas para uma diferença de $10 \%$ e de oito plantas para diferença de $20 \%$ (Zanon et al., 1997). Para as variáveis diâmetro do coleto e altura, o tamanho de amostra para Pinus taeda L., em viveiro 
florestal, variou em função da idade das plantas, que foram avaliadas de $15 \mathrm{em} 15$ dias (Silveira et al., 2009). Esses mesmos autores determinaram que o tamanho de amostra ideal para uma semiamplitude de $10 \%$ foi de 25 plantas para a altura e de 12 para o diâmetro do coleto.

Tendo em vista que não foram encontrados na literatura estudos sobre o dimensionamento de amostra para a avaliação de plantas de timbaúva, o objetivo deste trabalho foi estimar o tamanho de amostra (número de plantas) necessário para avaliar a altura de planta, o diâmetro do coleto e a relação altura de planta/diâmetro do coleto de plantas de timbaúva, e verificar a variabilidade do tamanho de amostra entre tubetes, doses de fertilizante e idades de avaliação.

\section{MATERIAL E MÉTODOS}

Foi conduzido um experimento em casa de vegetação com plantas de timbaúva (Enterolobium contortisiliquum). As sementes foram obtidas de frutos coletados em 16 árvores matrizes, localizadas em Santa Maria, Rio Grande do Sul. A coleta de sementes foi realizada entre final de maio e início de junho de 2009, com base nas recomendações de Sebbenn (2006), e posteriormente foram armazenadas em câmara fria $\left(8^{\circ} \mathrm{C}\right)$ e úmida (80\% UR) até a instalação do experimento em 20 de dezembro de 2009. Nesse momento, visando tornar a germinação uniforme, realizou-se um leve lixamento manual na parte oposta ao funículo de cada semente. Foram semeadas duas sementes em cada tubete, e 15 dias após foi feito o raleio, deixando uma planta por tubete.

Nesse experimento foram avaliados dez tratamentos, formados pela combinação de dois tamanhos de tubetes $\left(110\right.$ e $180 \mathrm{~cm}^{3}$ ) e cinco doses de fertilizante de liberação controlada (FLC) $\left(0,3,5,7\right.$ e $9 \mathrm{~g} \mathrm{~L}^{-1}$ de substrato). O substrato utilizado para o preenchimento dos tubetes foi o Carolina Soil ${ }^{\circledR}$ de baixa retenção, composto basicamente de turfa, vermiculita e casca de arroz carbonizada. O delineamento experimental foi em blocos ao acaso, com quatro repetições. As 40 unidades experimentais (parcelas) foram constituídas por bandejas de polipropileno com 24 tubetes, totalizando 960 tubetes no experimento.

A adubação de base foi realizada com FLC contendo $18 \% \mathrm{~N}$, sendo $9,5 \%$ e $8,5 \%$ nas formas amoniacal e de nitrato, respectivamente, $5 \%$ de $\mathrm{P}_{2} \mathrm{O}_{5}$ e $9 \%$ de $\mathrm{K}_{2} \mathrm{O}$, na forma granular, revestido com uma resina orgânica semipermeável. Conforme as especificações técnicas do fabricante, quando colocado em substrato úmido a uma temperatura média de $21,1^{\circ} \mathrm{C}$, a liberação de todos nutrientes ocorre no decorrer de 5 a 6 meses. A partir dos 21 dias após a semeadura até o final do experimento, foram realizadas, semanalmente, adubações de cobertura com o adubo Peter's Professional ${ }^{\circledR}$ (9-45-15) na dosagem de $5 \mathrm{~g} \mathrm{~L}^{-1}$, aplicado via solução aquosa por meio de pulverizador costal.

Aos 30, 60, 90, 120 e 150 dias após a semeadura (DAS), foi mensurada a altura de planta $(\mathrm{H})$, em centímetros, com régua milimetrada, e o diâmetro do coleto (D), em milímetros, com paquímetro digital, e calculada a relação altura de planta/diâmetro do coleto (HD), em seis plantas, tomadas aleatoriamente na área central de cada parcela. Os dados das características H, D e HD, em cada uma das cinco idades de avaliação, foram submetidos à análise de variância e ao teste $\mathrm{F}$ a $5 \%$ de probabilidade. As pressuposições de normalidade dos erros e homogeneidade das variâncias residuais foram atendidas. Para a análise de variância, foi utilizado o modelo matemático do delineamento em blocos com amostragem nas parcelas, definido em Storck et al. (2006).

A seguir, para os dados de $\mathrm{H}, \mathrm{D}$ e $\mathrm{HD}$ das seis plantas de cada uma das 40 parcelas, nas cinco idades de avaliação, foi verificada a normalidade, por meio do teste de Kolmogorov-Smirnov (Siegel \& Castellan, 2006), totalizando 600 testes ( 40 parcelas/avaliação $\times 5$ avaliações $\times 3$ características). A normalidade foi investigada a fim de verificar a adequação desses dados para o estudo do dimensionamento amostral, com base na distribuição t de Student.

Para as características H, D e HD, tomando-se por base as seis plantas amostradas em cada unidade experimental (parcela), de cada combinação de tamanho de tubete e dose de fertilizante, em cada idade de avaliação, foi calculado o tamanho de amostra (número de plantas, $\eta$ ) para as semiamplitudes do intervalo de confiança (erro de estimação) iguais a $1,2, \ldots, 10 \%$ da estimativa da média $(\mathrm{m})$, com grau de confiança (1-a) de 95\%, por meio da Expressão 1, conforme Bussab \& Morettin (2004):

$\eta=\frac{t_{\alpha / 2}^{2} s^{2}}{(\text { errode estimação })^{2}}$ 
Em que: $t_{\alpha / 2}$ é o valor crítico da distribuição t de Student, cuja área à direita é igual a $\alpha / 2$, isto é, o valor de $t$, tal que $P\left(t>t_{\alpha / 2}\right)=\alpha / 2$, com (n-1) graus de liberdade ( $\mathrm{n}=6$ plantas), com $\alpha=5 \%$ de probabilidade de erro, e s $\mathrm{s}^{2}$ é a estimativa de variância amostral.

Assim, obtiveram-se 30 variáveis (tamanho de amostra) formadas pela combinação das características $\mathrm{H}, \mathrm{D}$ e HD com os níveis de precisão de 1, 2, 3, 4, 5, 6, 7, 8, 9 e 10\% da média estimada, na unidade experimental. A fim de investigar a variabilidade do tamanho de amostra entre tubetes, doses e idades de avaliação, os dados dessas 30 variáveis (tamanho de amostra) foram submetidos à análise de variância considerando o modelo matemático do delineamento em blocos com parcelas subdivididas no tempo, definido em Storck et al. (2006).

Nesse modelo matemático proposto, os dez tratamentos (dois tamanhos de tubetes $\times$ cinco doses de fertilizante) e as cinco idades de avaliação formaram, respectivamente, a parcela principal e a subparcela no tempo. As médias do efeito principal de idades de avaliação foram comparadas por meio do teste de Scott-Knott (Ramalho et al., 2005) a 5\% de probabilidade. As análises estatísticas foram realizadas com o auxílio do programa SISVAR (Ferreira, 2011) e do aplicativo Office Excel.

\section{RESULTADOS E DISCUSSÃO}

Entre os 15 casos (três características $\times$ cinco idades de avaliação), por meio do teste $\mathrm{F}$ da análise de variância, verificou-se que houve interação tubete $x$ dose $(p \leq 0,05)$ em quatro casos (diâmetro do coleto aos 90, 120 e 150 dias após a semeadura e relação altura de planta/diâmetro do coleto aos 90 dias após a semeadura) (Tabela 1). Entre os demais 11 casos, em que não houve interação, os efeitos principais de tubete e de dose foram significativos em cinco e dez casos, respectivamente.

De maneira geral, em função dos efeitos significativos da interação tubete $\times$ dose e dos efeitos principais de tubete e de dose, pode-se inferir que há variabilidade entre as plantas, quanto às características altura de planta $(\mathrm{H})$, diâmetro do coleto $(\mathrm{D})$ e relação altura de planta/diâmetro do coleto (HD), nos distintos tamanhos de tubetes e doses de fertilizante de liberação controlada. Portanto, testes de comparações múltiplas de médias e análise de regressão seriam procedimentos complementares adequados, para a identificação do tamanho de tubete e da dose de fertilizante de liberação controlada, que proporcionariam plantas de timbaúva com desempenho superior em relação à $\mathrm{H}$, ao $\mathrm{D}$ e à $\mathrm{HD}$, até os 150 dias após a semeadura. No entanto, esses procedimentos não foram realizados por não ser o foco deste trabalho.

Em relação às características $\mathrm{H}, \mathrm{D}$ e $\mathrm{HD}$, o erro experimental (variação entre as parcelas com o mesmo tratamento) foi significativo $(\mathrm{p} \leq 0,05)$ na primeira idade de avaliação (30 dias após a semeadura). Também foi significativo em relação à $\mathrm{H}$ e HD, aos 60 e 120 dias após a semeadura, respectivamente. Nos demais dez casos, o efeito do erro experimental não foi significativo (Tabela 1).Com exceção da HD aos 120 dias após a semeadura, a variação entre as parcelas (erro experimental) foi maior que a variação entre as plantas dentro da parcela (erro amostral) nas primeiras avaliações. Assim, pode-se afirmar que para essas avaliações iniciais o procedimento adequado para melhorar a precisão experimental seria aumentar o número de repetições (Barbin, 2003; Ramalho et al., 2005; Storck et al., 2006).

Por outro lado, para as últimas avaliações (10 casos), o efeito do erro experimental não foi significativo. Nessa situação, aumentar o tamanho de amostra é considerado adequado para a melhoria da precisão experimental (Storck et al., 2006). Portanto, o redimensionamento do uso de quatro repetições (blocos) e, principalmente, a amostragem de seis plantas por parcela para avaliar as características $\mathrm{H}$, D e HD são convenientes para melhorar a precisão experimental. Particularmente neste estudo houve predominância de casos em que é adequado aumentar o tamanho de amostra.

O coeficiente de variação experimental (CVe), entre os 15 casos (três características $\times$ cinco idades de avaliação), oscilou entre 12,05\% e 22,65\%, e o coeficiente de variação amostral (CVa) variou entre 10,22\% e 18,39\% (Tabela 1). O CVe médio das cinco idades de avaliação foi $15,94 \%, 13,85 \%$ e $18,25 \%$, respectivamente, para as características H, D e HD. Ao passo que o CVa médio das cinco idades de avaliação foi relativamente inferior, ou seja, $12,78 \%, 11,87 \%$ e $15,26 \%$, respectivamente, para as características H, D e HD (Tabela 1).

As classes de precisão experimental, em função do coeficiente de variação experimental (CVe), foram propostas por Pimentel-Gomes (1990), o qual 
Tabela 1. Número de graus de liberdade (GL) e quadrado médio para as fontes de variação bloco, tubete, dose, tubete $\times$ dose, erro experimental e erro amostral, média, coeficiente de variação experimental (CVe) e coeficiente de variação amostral (CVa), para três características de plantas de timbaúva (Enterolobium contortisiliquum) avaliados em dez tratamentos (dois tamanhos de tubetes $\times$ cinco doses de fertilizante de liberação controlada) durante cinco idades de avaliação.

Table 1. Number of degrees of freedom (GL) and mean square to sources of variation block, tubes, dosages, tubes $\times$ dosages, experimental error and sampling error, average, coefficient of experimental variation (CVe) and coefficient of variation sampling (CVa), for three characteristics of timbaúva plants (Enterolobium contortisiliquum) valued at ten treatments (two sizes of tubes $\times$ five dosages of controlled release fertilizers) in five ages of evaluation.

\begin{tabular}{|c|c|c|c|c|c|c|}
\hline \multirow{3}{*}{ Fonte de Variação } & \multirow{3}{*}{ GL } & \multicolumn{5}{|c|}{ Idade de avaliação (dias após a semeadura) } \\
\hline & & 30 & 60 & 90 & 120 & 150 \\
\hline & & \multicolumn{5}{|c|}{ - } \\
\hline Bloco & 3 & $3,233^{\text {ns }}$ & $9,897^{\mathrm{ns}}$ & $17,995^{\star}$ & $14,301^{\mathrm{ns}}$ & $14,694^{\mathrm{ns}}$ \\
\hline Tubete & 1 & $69,230^{*}$ & $75,645^{*}$ & $28,154^{*}$ & $0,876^{\mathrm{ns}}$ & $5,828^{\text {ns }}$ \\
\hline Dose & 4 & $49,231^{*}$ & $192,409^{*}$ & $483,874^{*}$ & $687,522^{*}$ & $765,036^{*}$ \\
\hline Tubete $\times$ Dose & 4 & $2,968^{\text {ns }}$ & $8,351^{\mathrm{ns}}$ & $10,467^{\mathrm{ns}}$ & $15,464^{\mathrm{ns}}$ & $18,192^{\text {ns }}$ \\
\hline Erro experimental & 27 & $4,069^{\star}$ & $3,685^{\star}$ & $5,241^{\mathrm{ns}}$ & $6,967^{\mathrm{ns}}$ & $7,275^{\mathrm{ns}}$ \\
\hline Erro amostral & 200 & 1,465 & 2,083 & 3,468 & 5,444 & 7,011 \\
\hline Média & - & 11,21 & 13,15 & 15,18 & 16,45 & 16,90 \\
\hline CVe (\%) & - & 17,99 & 14,60 & 15,09 & 16,05 & 15,96 \\
\hline \multirow{2}{*}{ CVa (\%) } & - & 10,79 & 10,97 & 12,27 & 14,19 & 15,67 \\
\hline & & \multicolumn{5}{|c|}{ 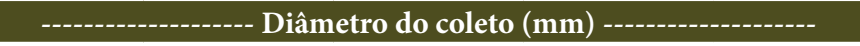 } \\
\hline Bloco & 3 & $0,091^{\mathrm{ns}}$ & $0,545^{\mathrm{ns}}$ & $0,942^{\mathrm{ns}}$ & $3,296^{*}$ & $1,573^{\text {ns }}$ \\
\hline Tubete & 1 & $0,000^{\text {ns }}$ & $0,233^{\text {ns }}$ & $2,546^{*}$ & $0,315^{\mathrm{ns}}$ & $5,260^{*}$ \\
\hline Dose & 4 & $0,932^{*}$ & $13,675^{\star}$ & $54,369^{*}$ & $127,380^{*}$ & $151,377^{\star}$ \\
\hline Tubete $\times$ Dose & 4 & $0,060^{\mathrm{ns}}$ & $0,530^{\mathrm{ns}}$ & $2,960^{*}$ & $3,010^{*}$ & $3,718^{*}$ \\
\hline Erro experimental & 27 & $0,119^{*}$ & $0,222^{\mathrm{ns}}$ & $0,575^{\mathrm{ns}}$ & $0,818^{\mathrm{ns}}$ & $0,869^{\text {ns }}$ \\
\hline Erro amostral & 200 & 0,073 & 0,214 & 0,395 & 0,557 & 0,672 \\
\hline Média & - & 2,65 & 3,91 & 5,15 & 6,07 & 6,39 \\
\hline CVe (\%) & - & 13,00 & 12,05 & 14,73 & 14,89 & 14,58 \\
\hline \multirow[t]{2}{*}{ CVa (\%) } & - & 10,22 & 11,83 & 12,21 & 12,29 & 12,82 \\
\hline & & \multicolumn{5}{|c|}{-------- Relação altura de planta/diâmetro do coleto --------- } \\
\hline Bloco & 3 & $0,956^{\mathrm{ns}}$ & $0,645^{\mathrm{ns}}$ & $0,846^{*}$ & $2,675^{\star}$ & $1,199^{*}$ \\
\hline Tubete & 1 & $9,972^{*}$ & $3,522^{*}$ & $0,099^{\text {ns }}$ & $0,027^{\mathrm{ns}}$ & $0,571^{\mathrm{ns}}$ \\
\hline Dose & 4 & $1,451^{\mathrm{ns}}$ & $0,948^{*}$ & $2,088^{*}$ & $4,999^{*}$ & $4,312^{*}$ \\
\hline Tubete $\times$ Dose & 4 & $0,167^{\mathrm{ns}}$ & $0,232^{\mathrm{ns}}$ & $0,747^{*}$ & $0,173^{\text {ns }}$ & $0,401^{\mathrm{ns}}$ \\
\hline Erro experimental & 27 & $0,680^{*}$ & $0,308^{\mathrm{ns}}$ & $0,205^{\text {ns }}$ & $0,404^{*}$ & $0,236^{\mathrm{ns}}$ \\
\hline Erro amostral & 200 & 0,281 & 0,217 & 0,194 & 0,228 & 0,254 \\
\hline Média & - & 4,26 & 3,40 & 2,99 & 2,80 & 2,74 \\
\hline CVe (\%) & - & 19,37 & 16,34 & 15,13 & 22,65 & 17,74 \\
\hline CVa (\%) & - & 12,44 & 13,72 & 14,72 & 17,02 & 18,39 \\
\hline
\end{tabular}

${ }^{\star}$ Efeito significativo pelo teste $\mathrm{F}$ em nível de $5 \%$ de probabilidade. ${ }^{\text {ns }}$ Não significativo.

estabeleceu que os experimentos com CVe inferior a $10 \%$, entre $10 \%$ e $20 \%$, entre $20 \%$ e $30 \%$ e superiores a $30 \%$ são classificados, respectivamente, como de alta, média, baixa e muito baixa precisão experimental. Assim, entre os 15 casos (três características $\times$ cinco idades de avaliação), em 1 (6,7\%) e em 14 (93,3\%) casos, os CVe foram enquadrados, respectivamente, nas classes de baixa e média precisão experimental.
Os experimentos com o menor coeficiente de variação experimental $(\mathrm{CVe}<10 \%)$, ou seja, com alta precisão experimental, devem ser almejados, e para isso o redimensionamento do tamanho de amostra é um procedimento apropriado.

Os maiores valores de CVe em relação ao coeficiente de variação amostral (CVa) evidenciam maior variabilidade das plantas entre as unidades experimentais do que 
dentro das unidades experimentais. Portanto, embora o aumento do tamanho de amostra seja eficiente, de maneira geral, também, aumentar o número de repetições contribui para melhorar a precisão experimental (Barbin, 2003). A característica HD apresentou maior valor médio de $\mathrm{CVa}$, o que comprova maior variabilidade entre as plantas dentro da parcela comparativamente à $\mathrm{H}$ e ao D. Isso sugere que, em plantas de timbaúva, para a estimação da média, com mesma precisão, o tamanho de amostra para as características $\mathrm{H}$ e D seja menor em relação à $\mathrm{HD}$, o que não representaria redução do trabalho a campo, já que HD é calculada em função de H e D. A variabilidade do tamanho de amostra entre características foi constatada em mudas de Eucalyptus saligna (Zanon et al., 1997), de Pinus taeda (Silveira et al., 2009) e de Cabralea canjerana (Cargnelutti Filho et al., 2012).

O valor p (nível mínimo de significância) do teste de Kolmogorov-Smirnov (Siegel \& Castellan, 2006), em relação aos dados das seis plantas, nos 600 casos analisados (40 parcelas/avaliação $\times 5$ avaliações $\times 3$ características), oscilou entre 0,186 e 0,999, e a média foi de 0,885. Os resultados do teste de Kolmogorov-Smirnov evidenciaram boa aderência dos dados de H, D e
HD à distribuição normal $(\mathrm{p}>0,185)$ e, portanto, são adequados para o estudo do dimensionamento amostral, com base na distribuição t de Student.

$\mathrm{Na}$ análise de variância dos tamanhos de amostra para a estimação da média das características H, D e $\mathrm{HD}$, não houve efeitos significativos da interação tripla (tubete $\times$ dose $\times$ idade), das interações duplas (tubete $\times$ dose, tubete $\times$ idade e dose $\times$ idade) e dos efeitos principais de tubete e de dose. Por sua vez, o efeito de idade de avaliação foi significativo ( $\mathrm{p} \leq 0,05)$ (Tabela 2).

O fato de as interações triplas e duplas serem não significativas revela que, em relação ao tamanho de amostra, os fatores tubetes, doses e idades de avaliação são independentes. Ainda, a constatação de que os efeitos principais de tubete e de dose também não foram significativos evidencia que não há variabilidade do tamanho de amostra entre tubetes e entre doses, ou seja, o mesmo número de plantas pode ser utilizado para avaliação dessas características nos diferentes tamanhos de tubete e doses de fertilizante. No entanto, o efeito significativo de idade de avaliação ( $\mathrm{p} \leq 0,05)$ revela que o tamanho de amostra para avaliar essas três características difere entre as idades de avaliação.

Tabela 2. Número de graus de liberdade (GL) e p-valor do teste F para as fontes de variação bloco, tubete, dose, tubete $\times$ dose, erro $_{1}$ (bloco $\times$ tratamento), idade, erro $_{2}$ (bloco $\times$ idade), tubete $\times$ idade, dose $\times$ idade, tubete $\times$ dose $\times$ idade

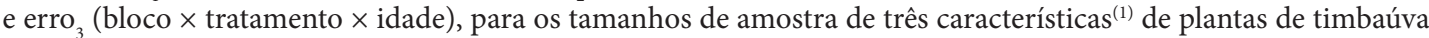
(Enterolobium contortisiliquum) avaliados em dez tratamentos (dois tamanhos de tubetes $\times$ cinco doses de fertilizante de liberação controlada) e em cinco idades de avaliação.

Table 2. Number of degrees of freedom (GL) and p-value of F-test to sources of variation block, tubes, dosages, tubes $\times$ dosages, error ${ }_{1}$ (block $\times$ treatment), age, error ${ }_{2}$ (block $\times$ age), tubes $\times$ age, dosages $\times$ age, tubes $\times$ dosages $\times$ age and error $_{3}$ (block $\times$ treatment $\times$ age), for sample sizes of three characteristics ${ }^{(1)}$ of timbaúva plants (Enterolobium contortisiliquum) valued at ten treatments (two sizes of tubes $\times$ five dosages of controlled release fertilizers) and five ages of evaluation.

\begin{tabular}{|c|c|c|c|c|}
\hline Fonte de Variação & GL & $\mathbf{H}$ & D & HD \\
\hline Bloco & 3 & 0,6115 & 0,3151 & 0,9549 \\
\hline Tubete & 1 & 0,7522 & 0,0549 & 0,8783 \\
\hline Dose & 4 & 0,8647 & 0,0812 & 0,6800 \\
\hline Tubete $\times$ Dose & 4 & 0,2092 & 0,1767 & 0,2443 \\
\hline Erro $_{1}($ Bloco $\times$ Tratamento $)$ & 27 & - & - & - \\
\hline Idade & 4 & 0,0000 & 0,0387 & 0,0074 \\
\hline Erro $_{2}($ Bloco $\times$ Idade $)$ & 12 & - & - & - \\
\hline Tubete $\times$ Idade & 4 & 0,9091 & 0,1438 & 0,4742 \\
\hline Dose $\times$ Idade & 16 & 0,1875 & 0,0586 & 0,8474 \\
\hline Tubete $\times$ Dose $\times$ Idade & 16 & 0,3961 & 0,2701 & 0,4940 \\
\hline Erro $_{3}($ Bloco $\times$ Tratamento $\times$ Idade $)$ & 108 & - & - & - \\
\hline
\end{tabular}

${ }^{(1)} \mathrm{H}=$ tamanho de amostra para a altura de planta, $\mathrm{D}=$ tamanho de amostra para o diâmetro do coleto e $\mathrm{HD}$ = tamanho de amostra para a relação altura de planta/diâmetro do coleto. 
Com isso, pode-se inferir que, nesse experimento, para a estimação da média das características H, D e HD, com mesma precisão, o tamanho de amostra (número de plantas) é único para os diferentes tubetes e doses e variável com as idades de avaliação. Para a avaliação do diâmetro do coleto e da altura de mudas de Pinus taeda, Silveira et al. (2009) também relataram que o tamanho de amostra variou em função da idade das plantas, e atribuíram as diferenças ao crescimento heterogêneo das plantas com o passar das avaliações. Também, para a avaliação de altura de planta, do diâmetro do coleto e da relação altura de planta/diâmetro do coleto de mudas de canjerana (Cargnelutti Filho et al., 2012), concluíram que o tamanho de amostra depende da idade de avaliação.

Entre as idades de avaliação, o tamanho de amostra (número de plantas) para a estimação da média das características $\mathrm{H}$, D e HD, foram formados três, dois e dois grupos, respectivamente, por meio do teste de
Scott-Knott (Tabela 3). Em relação à H, ao D e à HD, as três primeiras avaliações, ou seja, até os 90 dias após a semeadura, compuseram um grupo com menor variabilidade entre as plantas dentro da parcela, necessitando menor número de plantas para estimar a média com mesma precisão, quando comparadas às duas últimas idades de avaliação (120 e 150 dias após a semeadura), que apresentaram maior variabilidade entre as plantas (maior tamanho de amostra).

De maneira geral, o tamanho de amostra para a estimação da média de H, D e HD aumentou gradativamente com as idades de avaliação. O maior tempo de atuação do ambiente sobre essas últimas avaliações pode, provavelmente, explicar a maior variabilidade entre as plantas, imposta pela expressão gênica, já que o lote de sementes da timbaúva foi formado com o objetivo de restauração, ou seja, buscando abranger máxima variabilidade genética, conforme descrito em Sebbenn (2006). O aumento da variabilidade entre

Tabela 3. Tamanho de amostra (número de plantas) para avaliação da altura de planta, do diâmetro do coleto e da relação altura de planta/diâmetro do coleto de plantas de timbaúva (Enterolobium contortisiliquum), para as semiamplitudes do intervalo de confiança de $95 \%$ (erro de estimação) iguais a 1,2,.., $10 \%$ da estimativa da média, em cinco idades de avaliação (Idade), em dias após a semeadura.

Table 3. Sample size (number of plants) to evaluate height, collar diameter and relation height/collar diameter of timbaúva plants (Enterolobium contortisiliquum) for semiamplitudes confidence interval of 95\% (estimation error) equal to $1,2, \ldots, 10 \%$ of the estimated average, in five ages of evaluation (Age), at day after sowing.

\begin{tabular}{|c|c|c|c|c|c|c|c|c|c|c|}
\hline \multirow{3}{*}{ Idade } & \multicolumn{10}{|c|}{ 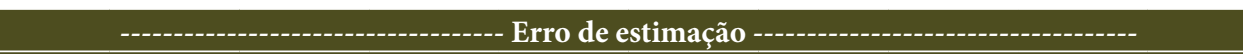 } \\
\hline & $1 \%$ & $2 \%$ & $3 \%$ & $4 \%$ & $5 \%$ & $6 \%$ & $7 \%$ & $8 \%$ & $9 \%$ & $10 \%$ \\
\hline & \multicolumn{10}{|c|}{ 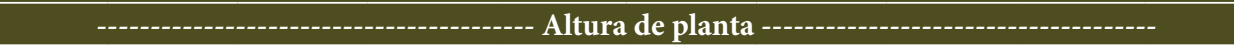 } \\
\hline 30 & 750 & 188 & 84 & 47 & 30 & 21 & 16 & 12 & 10 & $8 c^{*}$ \\
\hline 60 & 799 & 200 & 89 & 50 & 32 & 23 & 17 & 13 & 10 & $8 c$ \\
\hline 90 & 940 & 235 & 105 & 59 & 38 & 27 & 20 & 15 & 12 & $10 \mathrm{c}$ \\
\hline 120 & 1.184 & 296 & 132 & 74 & 48 & 33 & 25 & 19 & 15 & $12 \mathrm{~b}$ \\
\hline \multirow[t]{2}{*}{150} & 1.420 & 355 & 158 & 89 & 57 & 40 & 29 & 23 & 18 & $15 \mathrm{a}$ \\
\hline & \multicolumn{3}{|c|}{-------- } & \multicolumn{6}{|c|}{ - Diâmetro do coleto -- } & \\
\hline 30 & 692 & 173 & 77 & 44 & 28 & 20 & 15 & 11 & 9 & $7 \mathrm{~b}$ \\
\hline 60 & 920 & 230 & 103 & 58 & 37 & 26 & 19 & 15 & 12 & $10 \mathrm{~b}$ \\
\hline 90 & 1.044 & 261 & 116 & 66 & 42 & 29 & 22 & 17 & 13 & $11 \mathrm{~b}$ \\
\hline 120 & 1.272 & 318 & 142 & 80 & 51 & 36 & 26 & 20 & 16 & $13 \mathrm{a}$ \\
\hline \multirow[t]{2}{*}{150} & 1.216 & 304 & 136 & 76 & 49 & 34 & 25 & 19 & 16 & $13 \mathrm{a}$ \\
\hline & \multicolumn{10}{|c|}{ 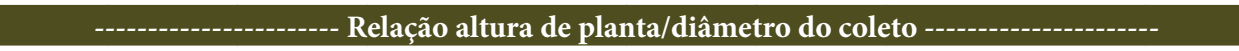 } \\
\hline 30 & 985 & 247 & 110 & 62 & 40 & 28 & 21 & 16 & 13 & $10 \mathrm{~b}$ \\
\hline 60 & 1.201 & 301 & 134 & 76 & 49 & 34 & 25 & 19 & 15 & $13 \mathrm{~b}$ \\
\hline 90 & 1.381 & 346 & 154 & 87 & 56 & 39 & 29 & 22 & 18 & $14 \mathrm{~b}$ \\
\hline 120 & 1.685 & 422 & 188 & 106 & 68 & 47 & 35 & 27 & 21 & $17 \mathrm{a}$ \\
\hline 150 & 2.131 & 533 & 237 & 134 & 86 & 60 & 44 & 34 & 27 & $22 \mathrm{a}$ \\
\hline
\end{tabular}

${ }^{*}$ Médias não seguidas de mesma letra diferem pelo teste de Scott Knott em nível de 5\% de probabilidade (aproximado). Nas colunas referentes aos erros de estimação de 1 a $9 \%$, as letras são as mesmas da coluna referente ao erro de estimação de $10 \%$, por isso não foram colocadas. 
as plantas dentro da unidade experimental com a idade das plantas, e, consequentemente, a necessidade de aumentar o tamanho de amostra para manter a precisão experimental, também tem sido constatado em mudas de pinus (Silveira et al., 2009) e de canjerana (Cargnelutti Filho et al., 2012).

O tamanho de amostra (número de plantas) para a estimação da média de cada característica, com semiamplitude do intervalo de confiança igual a $1 \%$ da estimativa da média (maior precisão) e coeficiente de confiança de $95 \%$, oscilou entre 692 plantas, para o diâmetro do coleto avaliado aos 30 dias após a semeadura, e 2.131 plantas, para a HD aos 150 dias após a semeadura (Tabela 3). Em outro extremo, ou seja, com semiamplitude do intervalo de confiança igual a 10\% da estimativa da média (menor precisão), o tamanho de amostra oscilou entre 7 e 22 plantas.

Os resultados deste estudo evidenciam que, do ponto de vista de técnicas experimentais, ao dimensionar o tamanho de amostra para uma avaliação precisa de plantas de timbaúva, as características e a idade das plantas devem ser consideradas, devido à variabilidade do tamanho de amostra entre as idades de avaliação e entre as características. Particularmente neste experimento, o número máximo de plantas por parcela (bandeja) foi limitado em 24 unidades. Então, estimativas de médias com altas precisões (1, 2, 3, 4 e 5\%) seriam impraticáveis, pois o tamanho de amostra é sempre superior a 24 plantas, enquanto estimativas com menor precisão (semiamplitude do intervalo de confiança igual a 10\%) são viáveis para as três características nas cinco idades de avaliação, pois o tamanho de amostra máximo seria de 22 plantas.

$\mathrm{Na}$ prática, as informações disponibilizadas possibilitam ao usuário dimensionar o tamanho de amostra conforme o número de plantas disponíveis para avaliação e a precisão desejada (erro de estimação máximo aceitável). Usando como exemplo este estudo, em que há 24 plantas para serem avaliadas, e tomando-se como referência o maior tamanho de amostra (relação altura de planta/diâmetro do coleto avaliada aos 150 dias após a semeadura, que corresponde à idade de expedição das mudas para plantio), pode-se inferir, com 95\% de confiança, que com 22 plantas o erro máximo na estimativa da média de $\mathrm{H}, \mathrm{D}$ e HD será de $\pm 10 \%$ da média estimada, independentemente do tubete, da dose e da idade de avaliação.

\section{CONCLUSÕES}

O tamanho de amostra (número de plantas) para avaliar a altura de planta, o diâmetro do coleto e a relação altura de planta/diâmetro do coleto de plantas de timbaúva (Enterolobium contortisiliquum) é dependente da idade de avaliação. Para os tamanhos de tubetes, doses de fertilizante de liberação controlada e idades de avaliação estudados neste experimento, 22 plantas por unidade experimental são suficientes para estimar a média de altura de planta, de diâmetro do coleto e de relação altura de planta/diâmetro do coleto de plantas de timbaúva, para um erro de estimação igual a $10 \%$ da média estimada, com grau de confiança de 95\%.

\section{STATUS DA SUBMISSÃO}

Recebido: 01, dez., 2014

Aceito: 22, nov., 2016

AUTOR(ES) PARA CORRESPONDÊNCIA

\section{Alberto Cargnelutti Filho}

Departamento de Fitotecnia, Centro de Ciências

Rurais, Universidade Federal de Santa Maria

- UFSM, Av. Roraima, s/n, Bairro Camobi, CEP 97105-900, Santa Maria, RS, Brasil

e-mail: alberto.cargnelutti.filho@gmail.com

\section{REFERÊNCIAS}

Araújo AP, Sobrinho SP. Germinação e produção de mudas de tamboril (Enterolobium contortisiliquum (Vell.) Morong) em diferentes substratos. Revista Árvore 2011; 35(3): 581-588. http://dx.doi.org/10.1590/S010067622011000400001.

Backes P, Irgang B. Árvores do sul: guia de identificação e interesse ecológico. Porto Alegre: Paisagem do Sul, 2009. $332 \mathrm{p}$.

Barbin D. Planejamento e análise estatística de experimentos agronômicos. Arapongas: Midas, 2003. 208 p.

Bussab WO, Morettin PA. Estatística básica. 5. ed. São Paulo: Saraiva, 2004. 526 p.

Cargnelutti Filho A, Araujo MM, Gasparin E, Avila AL. Dimensionamento amostral para avaliação de altura e diâmetro de mudas de Cabralea Canjerana. Ciência Rural 2012; 42(7): 1204-1211. http://dx.doi.org/10.1590/ S0103-84782012000700011. 
Carvalho PER. Espécies arbóreas brasileiras. Vol. 1. Brasília: EMBRAPA Informação Tecnológica; Colombo: EMBRAPA Florestas, 2003. 1039 p.

Ferreira DF. Sisvar: a computer statistical analysis system. Ciência e Agrotecnologia 2011; 35(6): 1039-1042. http:// dx.doi.org/10.1590/S1413-70542011000600001.

Gomes JM, Paiva HN. Viveiros florestais (propagação sexuada). 3. ed. Viçosa: UFV, 2004. 116 p. (Cadernos Didáticos, no. 72).

Gomes JM, Paiva HN. Produção de mudas de eucalipto por sementes. Informe Agropecuário 2008; 29(242): 14-22.

Haase D. Morphological and physiological evaluations of seedling quality. In: National Proceedings, Forest and Conservation Nursery Associations; 2007; Washington. Washington: USDA Forest Service Proceedings; 2007. p. 3-8.

Haase D. Understanding forest seedling quality: measurements and interpretation. Tree Planter's Notes 2008; 52(2): 24-30.

Jacobs DF, Landis TD. Fertilization. In: Dumroese RK, Luna T, Landis TD, editores. Nursery manual for native plants: a guide for tribal nurseries. Vol. 1. Washington: U.S. Department of Agriculture, Forest Service, 2009. p. 201215. (Nursery management. Agriculture Handbook, 730).

Luna T, Landis TD, Dumroese RK. Containers. In: Dumroese RK, Luna T, Landis TD, editores. Nursery manual for native plants: a guide for tribal nurseries. Vol. 1. Washington: U.S. Department of Agriculture, Forest Service; 2009. p. 95-111. (Nursery management. Agriculture Handbook, 730).

Oliet J, Segura ML, Dominguez FM, Blanco E, Serrada $\mathrm{R}$, Arias ML et al. Fertilizantes de liberacion controlada lenta aplicados a la produccion de planta forestal de vivero. Efecto de dosis y formulaciones sobre la calidad de Pinus halepensis mil. Investigación Agraria: Sistemas y Recursos Forestales 1999; 8(1): 207-228.

Pimentel-Gomes F. Curso de estatística experimental. 13. ed. Piracicaba: Nobel, 1990. 468 p.
Ramalho MAP, Ferreira DF, Oliveira AC. Experimentação em genética e melhoramento de plantas. Lavras: UFLA; 2005. 322 p.

Ritchie GA, Landis TD, Dumroese RK, Haase DL. Assessing plant quality. In: Landis TD, Dumroese RK, Haase DL. Seedling processing, storage and outplanting. Vol .7. Washington: U.S. Department of Agriculture, Forest Service; 2010. cap. 2, p. 17-81. (Agriculture. Handbook, 674).

Sebbenn AM. Sistemas de reprodução em espécies tropicais e suas implicações para a seleção de árvores matrizes para reflorestamentos ambientais. In: Higa AR, Silva LD. Pomar de sementes de espécies florestais nativas. Curitiba: FUPEF; 2006. 266 p.

Siegel S, Castellan NJ Jr. Estatística não-paramétrica para ciências do comportamento. 2.ed. Porto Alegre: Artmed; 2006. 448 p.

Silveira BD, Lúcio AD, Lopes SJ, Oliveira F. Aleatoriedade e tamanho da amostra em mudas de Pinus taeda L. Ceres 2009; 56(6): 730-735.

Storck L, Garcia DC, Lopes SJ, Estefanel V. Experimentação vegetal. Santa Maria: UFSM; 2006. 198 p.

Valeri SV, Corradini L. Fertilização em viveiros para produção de mudas de Eucalyptus e Pinus. In: Gonçalves JLM, Benedetti V. (Ed.). Nutrição e fertilização florestal. Piracicaba: IPEF; 2005. p. 167-190.

Wendling I, Guastala D, Dedecek R. Características físicas e químicas de substratos para produção de mudas de Ilex paraguariensis St. Hil. Revista Árvore 2007; 31(2): 209-220. http://dx.doi.org/10.1590/S0100-67622007000200003.

Zanon MLB, Storck L, Finger CA, Hoppe JM. Tamanho de amostra para experimentos de Eucalyptus saligna Smith em viveiro. Ciência Florestal 1997; 7(1): 133-138. http:// dx.doi.org/10.5902/19805098344. 